\title{
Identifying critical links on disruption-prone road networks: an approach that obviates scenario enumeration
}

\author{
Gopal R. Patil ${ }^{1 \%}$ and B. K. Bhavathrathan ${ }^{2}$ \\ ${ }^{1}$ Department of Civil Engineering, Indian Institute of Technology Bombay, Powai, Mumbai 400 076, India \\ ${ }^{2}$ Department of Civil Engineering, Indian Institute of Technology Palakkad, Palakkad 678 557, India
}

Road networks are susceptible to disruptions. Since disruptions on certain roads have graver impacts, critical link identification is vital in disruption preparedness. To identify critical links, most approaches systematically inject disruptions and evaluate the ensuing after-effects. Since degradation patterns are uncertain, such iterative approaches either presume knowledge of disruption patterns or try numerous patterns but soon escalate to computational impracticality on real-life road networks. This study obviates both, using a novel approach which measures the contribution of each link to a predefined operational limit on the network. It generates a criticality hierarchy on real-life networks, while on hypothetical symmetric networks it rightly identifies all links as critical.

Keywords: Critical links, disruptions, minimax optimization, road networks, scenario enumeration.

ROAD networks are susceptible to disruptions. Natural events (precipitation, waterlogging, etc.), human activities (incidents, repairs, etc.) and their combinations decrement the capacity of roadways. Disregarding the physical cause, they may be viewed as link disruptions on a network of node-to-link connections. Certain link disruptions cause graver impact than others and hence critical link identification is a vital problem in the design, planning and operation of road networks.

Popular perspectives in identifying critical links focus on network structure and behaviour ${ }^{1}$, where network functionality is commonly assessed in relation to the statistics of graph connectivity ${ }^{2}$, geodesic distances ${ }^{3,4}$, serviceability, usage data ${ }^{5}$, etc. These graph theoretic measures often fail to capture some principal characteristics of the network function ${ }^{6}$, such as disruptability and response to disruption. This could be a reason why, in his seminal work, Barabási ${ }^{7}$ opines that analyses must focus on linkdynamics, and think out of the conventional connectivitybased indices of network topology. On disruption-prone road networks, link capacity disruptions and their effect on link flows is an example of link dynamics.

*For correspondence. (e-mail: gpatil@civil.iitb.ac.in)
Dynamics in road networks is not link-specific. A disruption to one link has implications on flow, travel times and connectivity of a wider part of the network. Also, the impact of disruption on one particular link is quite different from that due to disruption on some other links. To identify the critical links, it is thus important to understand the significance of each link on the overall performance of the network. A popular approach to analyse this is to systematically assess the impact by disrupting individual links one after another ${ }^{8-14}$. However, this approach will not account for the interdependence of simultaneous disruptions on multiple links. One will have to enumerate every possible combination of multiple disruptions, and assess their impact scenario after scenario. Enumerating all possible scenarios of multiple disruptions is almost impossible on real-size road networks. As a solution to this issue, we propose a methodology which is conceptually reverse of the above-mentioned approach. We first identify a gravest possible impact - at which the network will still cater to the demand - of the disruptions. Subsequently, we assess which links have contributed most to the graveness, and label them as critical links.

The idea of altering (or removing) a link to study its effect on the transportation network was introduced by Halder $^{15}$. We refer to it as the iterative link removal strategy, which gained attention due to Scott et al. ${ }^{8}$, when they employed the approach to identify critical links of a network. Even though this involves link dynamics, this methodology is more suitable to studies related to the likes of computer and electrical networks, where the links usually get degraded totally. For transportation networks, the most likely cases are those of partial degradation. Sullivan et al. ${ }^{9}$, after observing that link removal is tantamount to fixing degradation level to $100 \%$, improved the method by employing different values of disruption. One can also come across other studies of this genredisrupting links iteratively and identifying which disruption has caused the maximum after-effect - in the literature ${ }^{10-14,16}$. Chen et al. ${ }^{17}$ observed that the strategy of iterative link removal will cause such computational burden, that applying this method on real-life networks will be impractical. As an alternative, they suggested defining the impact area for each link of the network, and 


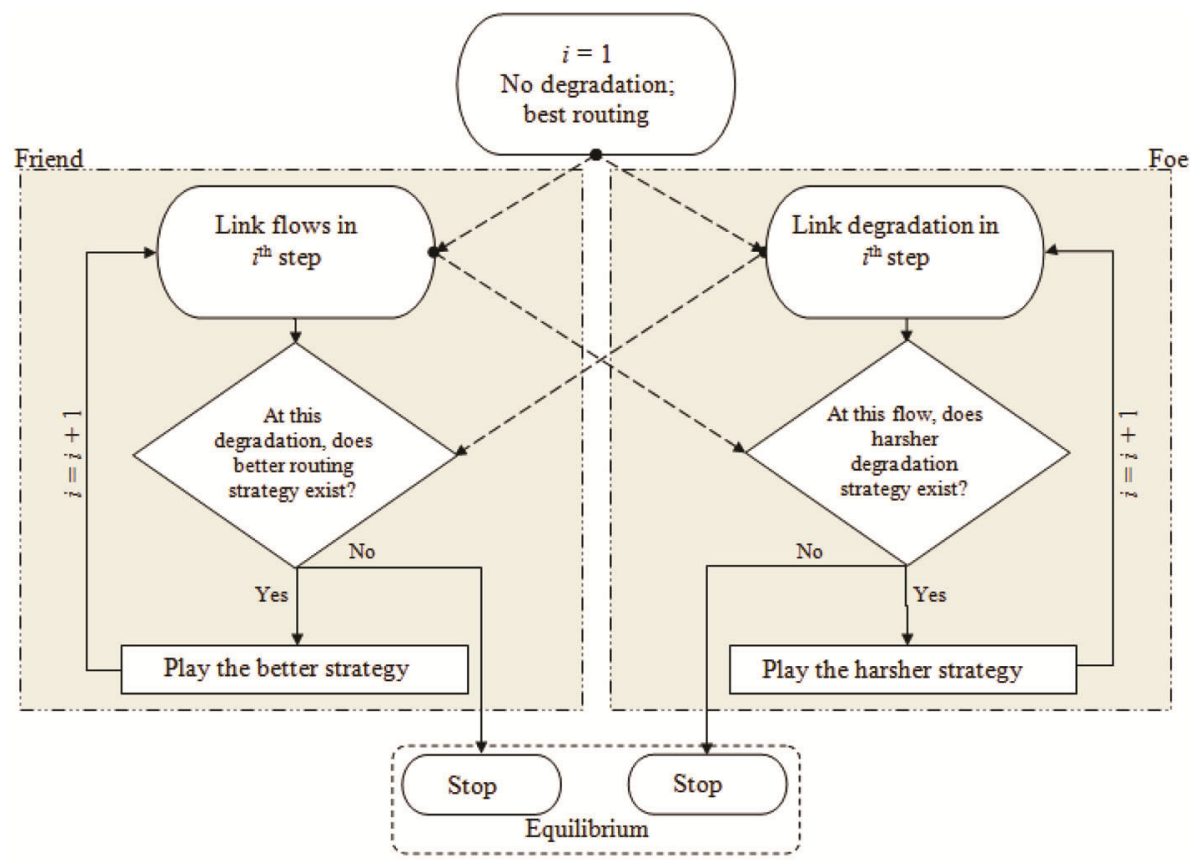

Figure 1. Schematic of the game.

analysing the effect limited to that specific area ${ }^{17}$. A review of such different iterative link removal methods and their quality has been reported by Knoop et al. ${ }^{18}$, who conclude that most of them are not self-sufficient in quantifying link criticality, and their combinations are also not satisfactory.

As discussed, road networks are subject to multiple disruptions, simultaneously on different links. In the abovediscussed studies that employ iterative removal $1^{8,9,11-14}$, other links of the network are deemed to be non-disrupted during removal of one (a set of) $\operatorname{link}(\mathrm{s})$; whereas in the case of game theoretic models, predefined discrete scenarios are considered ${ }^{19,20}$. Either way, the after-effect of network degradation is under-estimated, since all scenarios that arise out of various combinations of multiple simultaneous disruptions are not accounted exhaustively. To account this, the above methods have to be repeated with different levels of degradation on different (sets of) degraded link(s). Such a relaxation attracts what is known as the curse of dimensionality. For instance, a network with 100 links, assuming only $100 \%$ and $0 \%$ capacity realization states, would cause $2^{100}$ scenarios ( $n$ links, each with $k$ levels of degradation cause $k^{n}$ scenarios).

Here, a different approach is presented, which allows continuous capacity variability on all links of the network, thus obviating disruption-scenario enumeration. It is thus feasible to identify the gravest possible operable after-effect on the network, and analyse which links have contributed to it. The link that contributes most to such a scenario is the most critical link. The next section details the definition and identification of such scenarios by solving what we call the critical state problem, and using it, establish the method to identify critical links.

\section{Critical state and its applicability in identifying critical links}

\section{The critical state problem}

Recently, a critical state of road networks has been defined with the method to compute it - called the critical network state problem (CSP)-being formulated in deterministic and stochastic set-ups, and applied in measuring network resilience ${ }^{21-23}$. To find the critical links of a network, we solve the deterministic version of the $\mathrm{CSP}^{21}$. The problem envisages a two-player non-cooperative game between two imaginary players: a friend and a foe of the network. The friend tries to minimize and the foe tries to maximize the system travel time (STT). The foe degrades the capacity of multiple links simultaneously to increase the STT, while the friend reroutes the traffic to decrease it. Either player is aware of the other's decision, and will alter strategies until an equilibrium is reached. At this equilibrium, the network will be in a critical state: one in which neither of the players can betteroff their objectives by unilaterally switching the strategies. Figure 1 is a schematic presentation of the game. We emphasize that the players need not be realistic agents, as the goal of CSP is to determine an abstract state of the network at which it will operate at a cost which is irreducible, even by adopting the best possible routing ${ }^{21,22}$. 
Disruption on one or more links increases the operation cost on the network. While the network operation cost increment can take multiple forms, such as emissions, vehicle miles, monetary, accident risks, etc. this study accounts for network cost using its most popular proxy, i.e. STT. Therefore, the link criticality discussed here is directly in terms of travel time. However, one may redefine criticality with respect to other implications of disruption, and aptly reformulate the objective function in the CSP. Thereby, the methodology being proposed can be transferred across multiple narratives of criticality. However, while doing so, one must pay attention on the ensuing structure of such reformulations.

After solving the CSP, critical links are identified based on the contribution to network cost increment by each link. These links are the major contributors towards the critical state of the network. The idea is that the imaginary attacker will prefer certain links that increment the network cost. Even though this idea of using game theory to assess criticality is relatively fresh in the area of road networks, similar approaches can be seen in defence policies, controlling drug trafficking, etc. ${ }^{24,25}$. In studies related to transportation networks, some researchers have used game theoretic models to assess vulnerability ${ }^{19,20}$, and optimize protection ${ }^{26}$. While the methodology by Murray-Tuite and Mahmassani ${ }^{19}$ applies limited degradation to a subset of links, Bell et al. ${ }^{20}$ invoke a scenario analysis with predefined degradation levels. CSP relaxes such assumptions, as can be seen in the next section, which briefly presents its formulation.

\section{Problem formulation}

Road network is considered as a digraph $G(N, A)$, where $N$ is the set of nodes and $A$ is the set of directed links. $R$ denotes the set of origins and $S$ denotes the set of destinations. The vector $\boldsymbol{D}$ denotes the degraded capacities, and $\boldsymbol{X}$ denotes the flows on each link of the set $A . A_{i+}$ and $A_{i-}$ respectively denote the set of outgoing links and set of incoming links at node $i$. Table 1 gives the other notations associated with the formulation.

CSP is formulated as a minimax optimization problem $^{21}$, which finds the minimum possible value of a systemic cost function with respect to one set of decision

Table 1. Notations used in the critical network state problem (CSP)

\begin{tabular}{ll}
\hline$Q_{r s}$ & Demand between the origin $r$ and destination $s ;$ \\
& $(r \in R ; s \in S)$. \\
$i j$ & Directed link between nodes $i$ and $j ; i j \in A$. \\
$x_{i j}$ & Flow on link $i j ; x_{i j} \in X$. \\
$f_{i j}^{s}$ & Flow specific to destination $s$ on link $i j$. \\
$K_{i j}$ & Base capacity on link $i j$. \\
$y_{i j}$ & Divisive factor; $y_{i j} \in Y: 1 / y_{i j}$ is the realized proportion \\
& of $K_{i j}$. \\
$c_{i j}\left(x_{i j}, y_{i j}\right)$ & Link cost function \\
\hline
\end{tabular}

variables (link flows), simultaneously finding the maximum possible value of the same function with respect to a different set of decision variables (link degradation). The formulation is reproduced below.

$$
z=\min _{x_{i j}} \max _{y_{i j}} \sum_{i j} x_{i j} \cdot c_{i j}\left(x_{i j}, y_{i j}\right) .
$$

Subject to

$$
\begin{aligned}
& \sum_{i j \in A_{i^{+}}} f_{i j}^{s}-\sum_{i j \in A_{i^{-}}} f_{j i}^{s}=q^{i s}, \forall i, i \neq s, \\
& \sum_{s} f_{i j}^{s}=x_{i j}, \quad \forall_{i j}, \\
& 0 \leq x_{i j} \leq\left(K_{i j} / y_{i j}\right), \quad \forall_{i j}, \\
& 1 \leq y_{i j} \leq m_{i j}, \forall_{i j},
\end{aligned}
$$

where $m_{i j}$ is an upper bound on $y_{i j}$ set by the analyst. Such upper bounds are not formal requirements, but by doing so, $1 / m_{i j}$ will be the minimum proportion of capacity that stays. If $m_{i j}$ is set as 100 , it imparts a limit of $99 \%$ on link capacity degradation. In the absence of information on degradation pattern, one can use a very high value of $m_{i j}$, thereby enabling total degradation for practical reasoning. On the other hand, the analyst may also opt to remove some links from getting degraded, or limit degradation on certain unlikely links, if such data are available.

In the above formulation, a common form of link-cost function as given in eq. (6) is used. $T_{i j}$ is the free-flow travel time on link $i j$, while $\alpha$ and $\beta$ are model parameters.

$$
c_{i j}\left(x_{i j}, y_{i j}\right)=T_{i j}+\beta\left(\frac{x_{i j}}{K_{i j} / y_{i j}}\right)^{\alpha} .
$$

The base requirement for the usual minimax theorem

$$
\left(\min _{y \in Y} \max _{x \in X} f(x, y)=\max _{x \in X} \min _{y \in Y} f(x, y)\right)
$$

is that we use an unconstrained zero-sum game. Since CSP is a constrained game, the usual minimax theorem will not straightforwardly hold. Nevertheless, as eq. (6) is a monotonously increasing convex cost function (with respect to the variable set $X$ only), based on Fan's generalization of the minimax theorem ${ }^{27}$, minimax theorem holds on CSP. The minimax value will hence be an equilibrium, and $z$ in eq. (1) is the equilibrium payoff of the zero-sum game between a minimizer and a maximizer. 
Such a game has a unique payoff, and thus the value of $z$ is unique ${ }^{28}$. Even though the objective function value is unique at every solution, the decision variables need not be, as established below. In spite of the uniqueness on payoff, CSP is non-convex and hard to solve. We thus employ a fictitious play algorithm for solving it on this sample network, and modify it further for solving realistic networks.

To ascertain the existence of equal-payoff multiple equilibria, i.e. multiple solutions with the same value of objective function, we adopt the fictitious play algorithm $(\mathrm{FPA})^{29}$. In each round of the fictitious play, either player responds with the best possible strategy to the accumulated observations from the other player's previous moves. It is known that the FPA will converge to an equilibrium in two-player zero-sum games with finite strategies $^{30}$. Figure 2 presents the sample network on which FPA is employed. $T_{i j}$ is assumed to be 5 units, and no binding restriction of $m_{i j}$ is kept on $y_{i j}$ in this case. A demand of six units from node 1 to node 4 is considered. All links have the capacity of 12 units. The $\alpha$ and $\beta$ values for eq. (6) are taken as 4 and 0.15 respectively. Table 2 shows two resulting equilibria. Note that, in spite of different values on the decision variables, the value of the objective function (STT) is same in both the equilibria.

\section{Link criticality}

Definition. Link criticality (LC) is defined as the relative contribution that the link makes to the difference of STT at the critical state and base state (eq. (7)).

$$
\mathrm{LC}_{i j}=\frac{x_{i j}^{*} c_{i j}\left(x_{i j}^{*}, y_{i j}^{*}\right)-x_{i j}^{b} c_{i j}\left(x_{i j}^{b}, 1\right)}{\sum_{i j} x_{i j}^{*} c_{i j}\left(x_{i j}^{*}, y_{i j}^{*}\right)-\sum_{i j} x_{i j}^{b} c_{i j}\left(x_{i j}^{b}, 1\right)} \forall i j,
$$

where $x_{i j}^{*}$ is the flow on link $i j$ at the critical state, $y_{i j}^{*}$ the degradation factor at the critical state and $x_{i j}^{b}$ is the system-optimal flow at the non-disrupted network state.

As discussed, the solution of CSP is not unique. Thus, multiple solutions of the problem may result in different

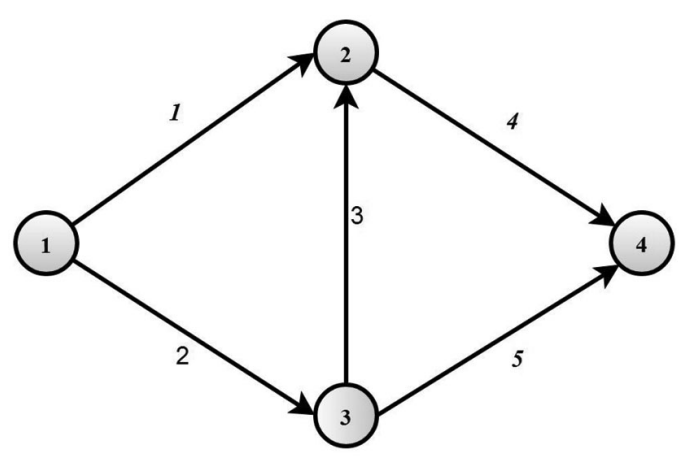

Figure 2. Sample network to ascertain the existence of equal-payoff multiple equilibria. values of $x_{i j}$ and $y_{i j}$, and subsequently in different values of LC. We propose the use of average criticality $\overline{\mathrm{LC}}$, which is obtained by solving CSP multiple times and is given by eq. (8).

$$
\overline{\mathrm{LC}}_{i j}=\frac{\sum_{m=1}^{M} L C_{i j}^{m}}{M} \forall i j,
$$

where $L C_{i j}^{m}$ is the link criticality of link $i j$ in $m$ th solution and $M$ is the number of times the problem is solved. $x_{i j}^{b}$ can be found from the standard system-optimal routing at zero-degrataion $^{31}$, and is not detailed here. $x_{i j}^{*}$ and $y_{i j}^{*}$ are obtained by solving CSP.

An immediate question of practical interest follows, concerning the value of $M$ in eq. (8). While this number might vary depending on the size of the network and the demand profile, one may resort to tools generally employed in traffic simulation studies to arrive at an indicative value. For instance, one method to estimate the number of repetitions required in discrete event simulations can be determined by a two-step approach ${ }^{32}$. First, estimate the standard deviation of outputs after performing an arbitrary number (say, $M_{0}$ ) of repetitions. In our case, the output will be the vector of expected link criticality. Assuming that such an output would converge as the number of repetitions increase, the following number of repetitions as suggested by Fishman $^{32}$ can be performed

$$
M=\left[\frac{\sigma_{M_{0}}(O) t_{\alpha / 2}}{\varepsilon_{M}}\right]^{2},
$$

where $\sigma_{M_{0}}(O)$ is the standard deviation of the output $O$ from $M_{0}$ repetitions, $t_{\alpha / 2}$ is the critical value of the $t$-distribution at significance level $\alpha$ and $\varepsilon_{M}$ is the target allowable error after $M$ repetitions.

\section{Modified fictitious play algorithm to solve CSP}

The non-convexity of CSP makes it hard to solve, and requires the use of a specific algorithm to solve it on bigger networks. A modified version of the FPA mentioned in previous section is employed. As a detailed description of this algorithm is available elsewhere ${ }^{33}$, we provide a brief outline here for the sake of continuity in reading.

In the modified fictitious play - known as the weighted fictitious play (WFP) - players' beliefs are updated by depreciating the strategies that the opposite player used before a few rounds. In effect, the players weigh the opponent's recent actions more ${ }^{34}$, as opposed to the basic fictitious play where all previous actions are equally weighed. In other words, an instance of WFP with equal placements of weights on each of the previous steps 
Table 2. Evidence of existence of multiple equilibria with the same payoff; results using fictitious play algorithm on the sample network

\begin{tabular}{|c|c|c|c|c|c|c|}
\hline \multirow[b]{3}{*}{$\begin{array}{l}\text { Equilibrium } \\
\text { no. }\end{array}$} & \multirow[b]{3}{*}{ Link } & \multicolumn{4}{|c|}{ Values of decision variables } & \multirow[b]{3}{*}{$\begin{array}{l}\text { System travel time } \\
\text { (STT) at equilibrium }(z)\end{array}$} \\
\hline & & \multicolumn{2}{|c|}{ At initial point } & \multicolumn{2}{|c|}{ At equilibrium } & \\
\hline & & $\begin{array}{c}\text { Flow } \\
\left(x_{i j}\right)\end{array}$ & $\begin{array}{l}\text { Disruption } \\
\text { factor }\left(y_{i j}\right)\end{array}$ & $\begin{array}{c}\text { Flow } \\
\left(x_{i j}\right)\end{array}$ & $\begin{array}{l}\text { Disruption } \\
\text { factor }\left(y_{i j}\right)\end{array}$ & \\
\hline \multirow[t]{5}{*}{1} & 1 & 0.00 & 1.00 & 3.00 & 4.00 & 61.8 \\
\hline & 2 & 0.00 & 1.00 & 3.00 & 4.00 & \\
\hline & 3 & 0.00 & 1.00 & 0.00 & 1.00 & \\
\hline & 4 & 0.00 & 1.00 & 3.00 & 4.00 & \\
\hline & 5 & 0.00 & 1.00 & 3.00 & 4.00 & \\
\hline \multirow[t]{5}{*}{2} & 1 & 0.00 & 4.75 & 1.66 & 7.25 & 61.8 \\
\hline & 2 & 6.00 & 2.00 & 4.34 & 2.76 & \\
\hline & 3 & 0.00 & 9.82 & 0.00 & 9.82 & \\
\hline & 4 & 0.00 & 5.63 & 1.66 & 7.25 & \\
\hline & 5 & 6.00 & 2.00 & 4.34 & 2.76 & \\
\hline
\end{tabular}

throughout the history of the play, will result in the basic FPA. In this sense, WFP may be called a generalized version of the FPA. WFP is interpreted as a process in which the players have limited memory, and forget the opponent's actions beyond a certain number of plays. This interpretation intuitively explains how WFP relaxes the issues pertaining to memory and computational complexity while dealing with large games. The players only need to keep track of the opponent's latest $N$ actions, and can discard those before $N$ steps. As the convergence to equilibrium would require a large number of iterations, the option of discarding historic information eases the computational requirements.

Computing the best response is the vital part of the WFP algorithm. In typical finite games, computing best response is just a standard linear program ${ }^{35}$, as in such games there exists a cost vector $c$ for each player such that $c \times x$ explicitly defines the payoff associated with strategy $x$. However, the cost function of the friend-foe game is continuous in nature and the responses are bound by a set of constraints as described in eqs (1)-(5). Hence, computing the best response is not as straightforward as in typical matrix form games.

However, in a routing perspective, the best response at any instant is a flow pattern that minimizes the cost. Since the cost is modelled as STT, it follows that the best response is the system-optimal flow pattern. Thus, a traditional method - the Frank-Wolfe (FW) algorithm - is employed to obtain the system-optimal solution. It is known that the solution of any user-equilibrium program with marginal link travel times would produce a systemoptimal flow pattern ${ }^{31}$. Marginal travel time is the marginal contribution to link travel time from an additional infinitesimal flow. As a result, at an equilibrium of marginal travel times, no link would gain a reduction in travel time by reassigning some of its flows to another link. This property is harnessed in obtaining the systemoptimal flow pattern using the process.
The formulation is solved on topologically and parametrically symmetric, and asymmetric networks. While some links can be expected to be more critical on asymmetric networks, all links will be equally important on symmetric networks.

\section{Numerical examples}

\section{Symmetric test networks}

The methodology presented here is first illustrated on symmetric test networks. By symmetric network, we mean that both the supply and demand sides of the road network are symmetrical. In other words, both the geometry and origin-destination matrix are symmetric. For instance, on a network with only two nodes $A$ and $B$, the links connecting $A$ to $B$ and back must have the same lengths or free-flow travel times. Both of these links must follow the same cost function and have the same capacities. Also, both must act as origin and destination simultaneously, and the travel demand from $A$ to $B$ must be the same as that from $B$ to $A$.

On such symmetric networks, we can expect all links to have equal criticality, of how criticality is defined, and of the method employed to compute it. Therefore, we can use the method on symmetric networks as control experiments to validate it. We thus use our method on two such networks as shown in Figure 3. Five nodes act as origins and destinations with equal demand from each node to all other nodes. These networks have such topologies, link attributes and demand matrices that they make the network parametrically and topologically symmetric. The inter-zonal demand is assumed consistently as 250 across all zones for both the networks in Figure 3. The five zones of both the test networks are geographically the same and the two networks are topological options in which the five zones can be symmetrically connected. 


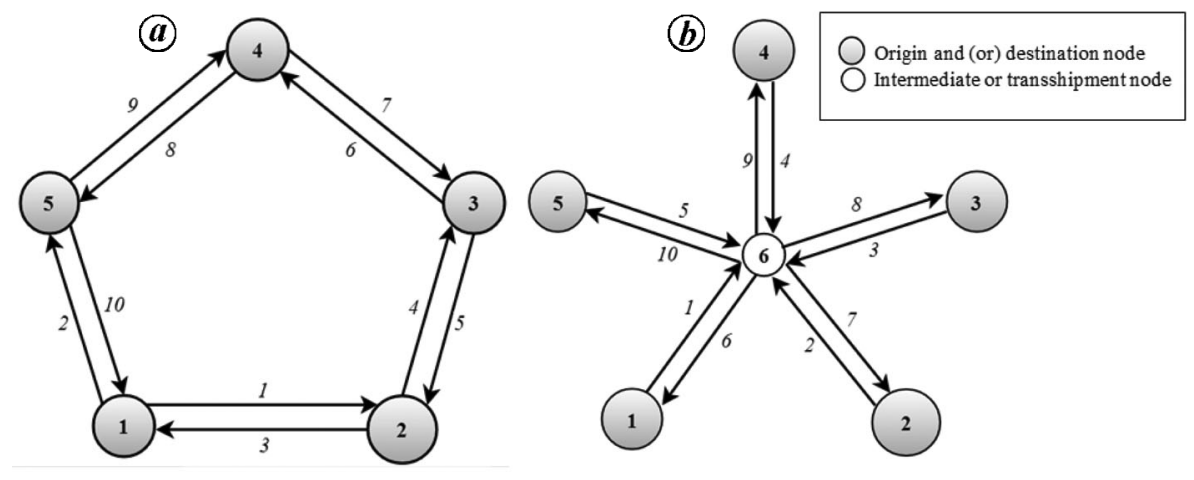

Figure 3. $\boldsymbol{a}, \boldsymbol{b}$, Symmetric test networks 1 and 2 .

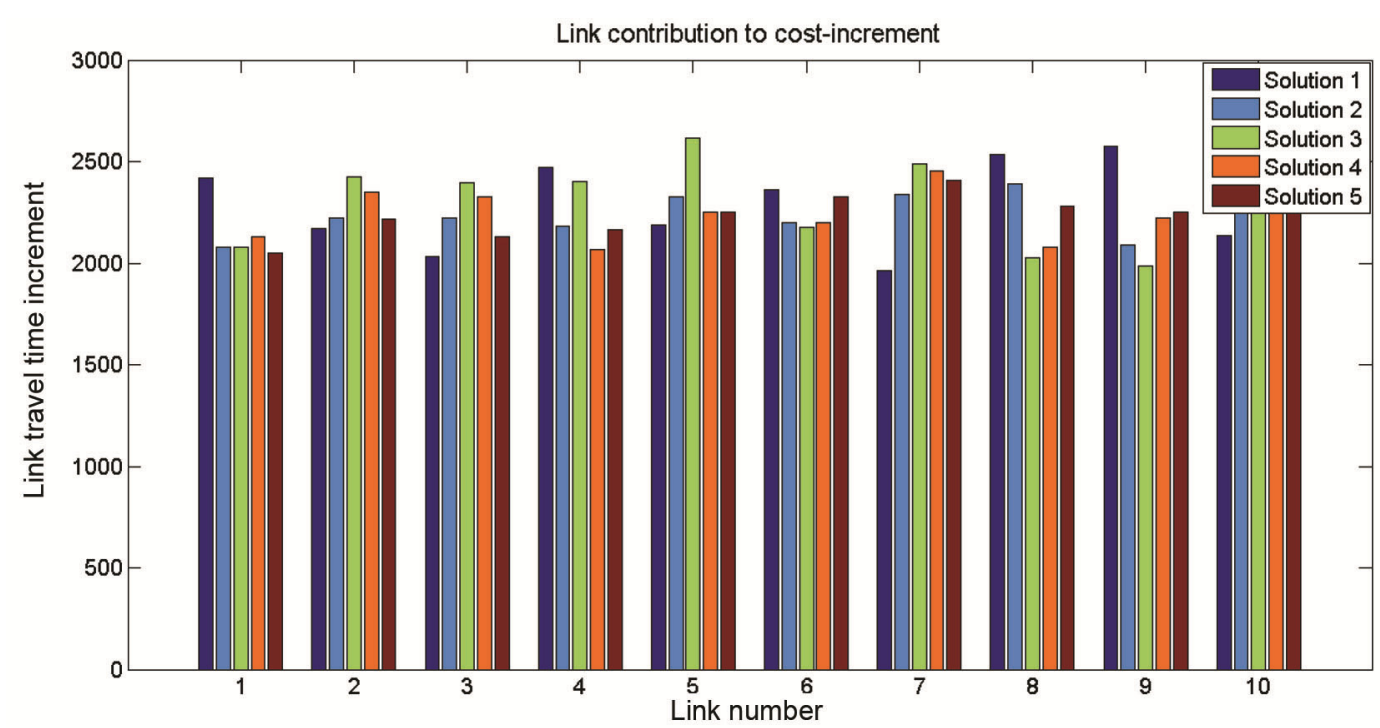

Figure 4. Non-uniqueness of decision variable on symmetric test network 1 .

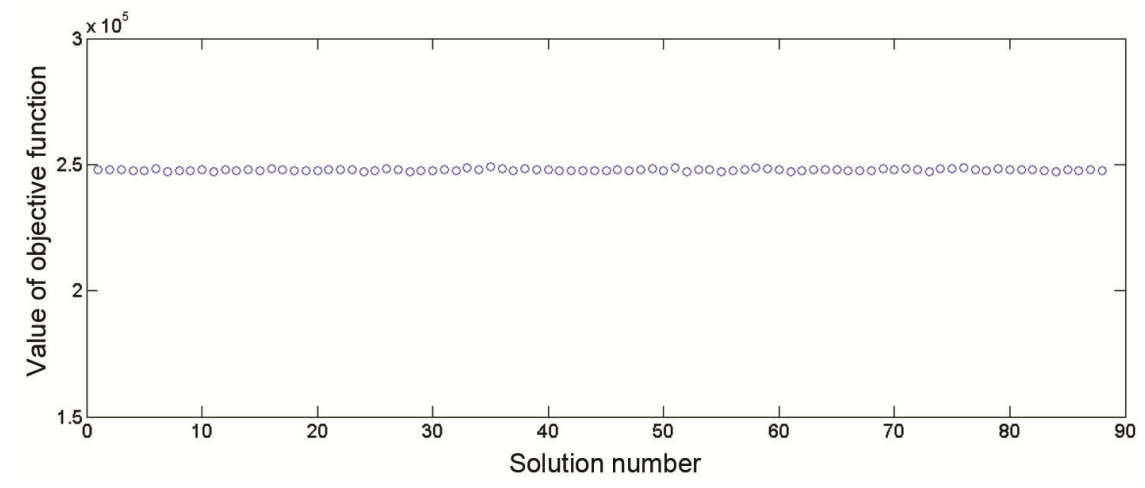

Figure 5. Uniqueness of objective function on symmetric test network 1 .

After obtaining one solution for CSP, LC values are found using eq. (7). The non-uniqueness of solution is evident from the link travel times in Figure 4. Recall that only the decision variables will be non-unique. The value of the objective function will be unique at every solution. This is evident from Figure 5, where the relative standard deviation (RSD) is only $0.16 \%$, which is due to the tolerance of convergence criterion. The $\overline{\mathrm{LC}}$ values obtained using eq. (8), are found to be comparable on all the ten links of the symmetric test networks 1 and 2 , as can be seen from Figures 6 and 7. The RSD of $\overline{\mathrm{LC}}$ across all links in the symmetric transportation network 1 is $0.005 \%$ and that in the symmetric transportation network 2 is $0.032 \%$, which indicates that the methodology finds all links to be similarly critical on symmetric test networks, as expected. 

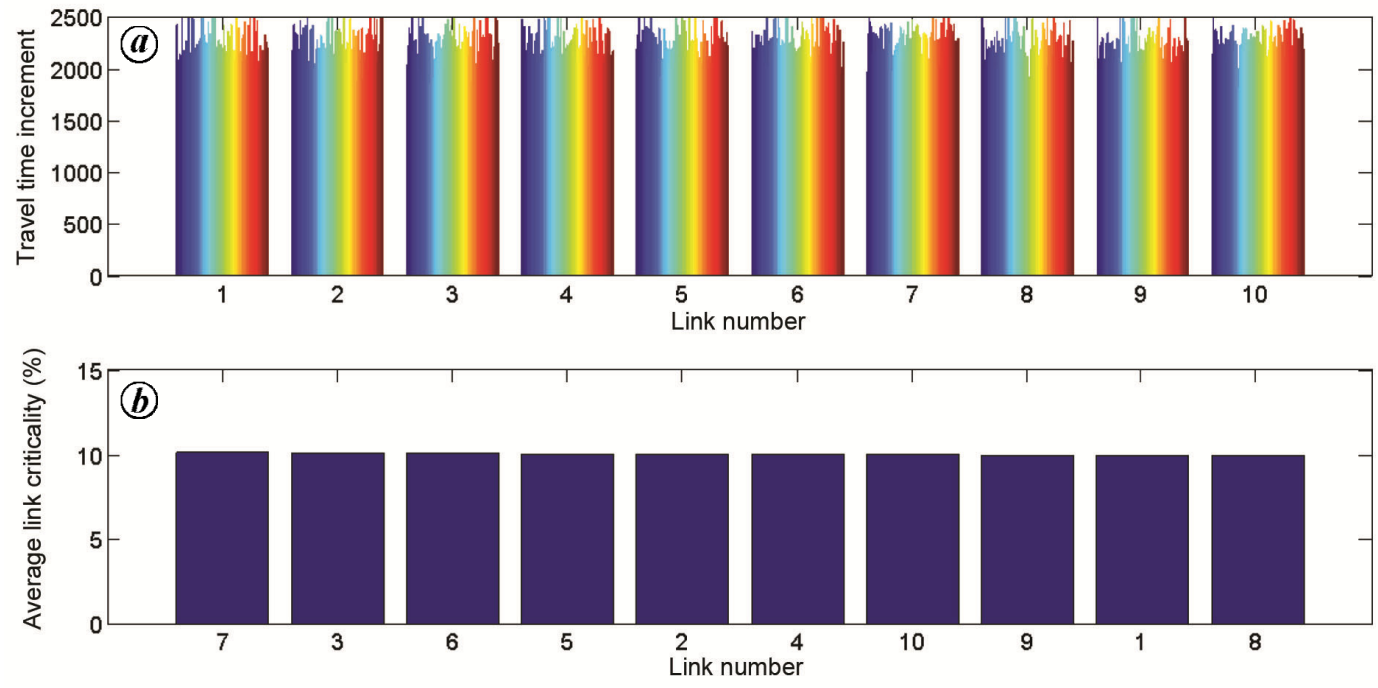

Figure 6. Critical links of the symmetric test network 1. $\boldsymbol{a}$, Link contribution to cost increment. $\boldsymbol{b}$, critical links in descending order of rank.
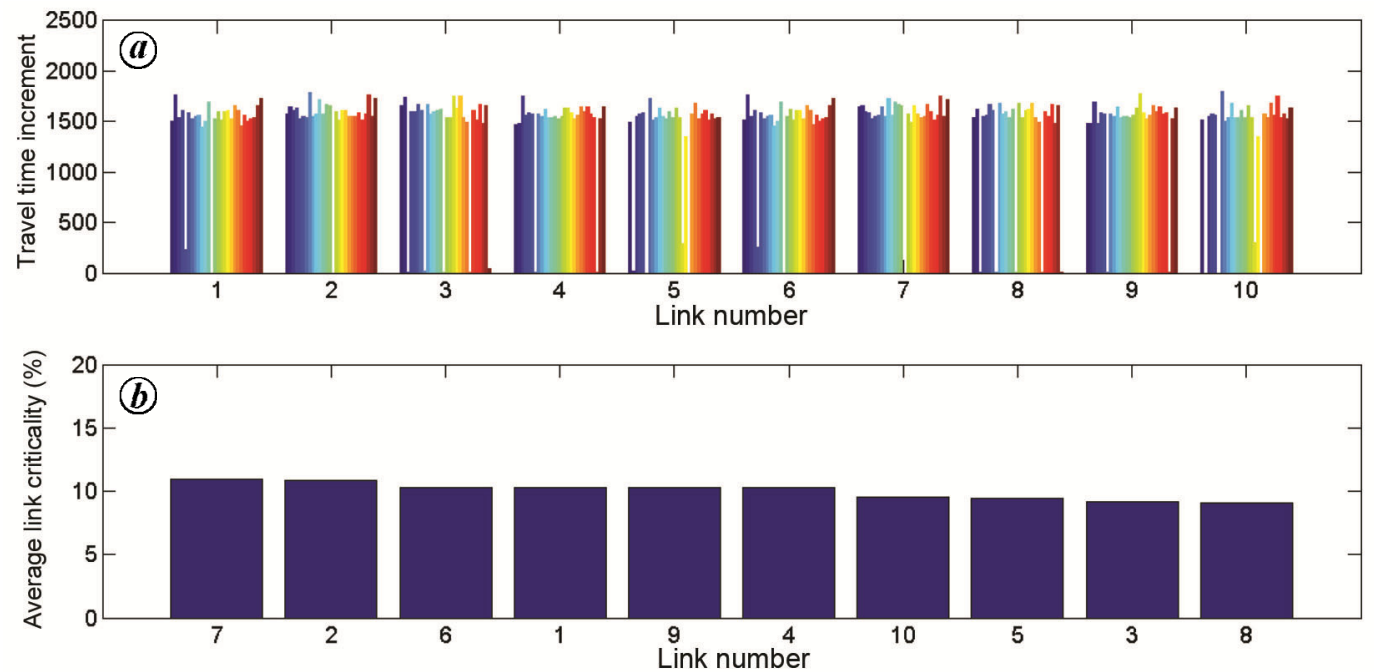

Figure 7. Critical links of the symmetric test network 2. $\boldsymbol{a}$, Link contribution to cost increment. $\boldsymbol{b}$, critical links in descending order of rank.

\section{Asymmetric test networks}

The numerical examples on symmetric networks mentioned in the previous section emphasize the validity of the method presented here. Subsequently, this section provides results pertaining to networks that are popularly adopted by researchers to exemplify network analyses. Needless to mention, these networks are asymmetric as the interest here is to perform analyses on different topologies that have some flavour of realistic transportation networks. Figure 8 shows these networks.

The CSP formulation was solved on the three asymmetric test networks, and LC was calculated using eq. (7). The attributes of the asymmetric test networks 1-3 can be found in Sheffi ${ }^{31}$, Harker and Friesz ${ }^{36}$, Nguyen and
Dupuis ${ }^{37}$ respectively, from which they are adopted. Table 3 shows the result on the asymmetric test network 3 at a single trial. Link 14 is the most critical link, followed by links 2, 11 and 7 respectively. Note that at this solution, link 18 shows a decrease in its contribution to STT at the critical state. This is because while the capacity is not much degraded on that link, the flow has decreased. A possible reason for decrease in flow can be high degradation on the adjacent links. In this case, while link 18 undergoes a decrease in flow, its adjacent links 2 and 11 (Figure 8) undergo high degradation. It is thus possible that some links end up having negative value for LC. Since the solution of the minimax optimization problem need not be unique, the experiment was performed 100 times. Figure $9 a$ shows the results from all performances. 

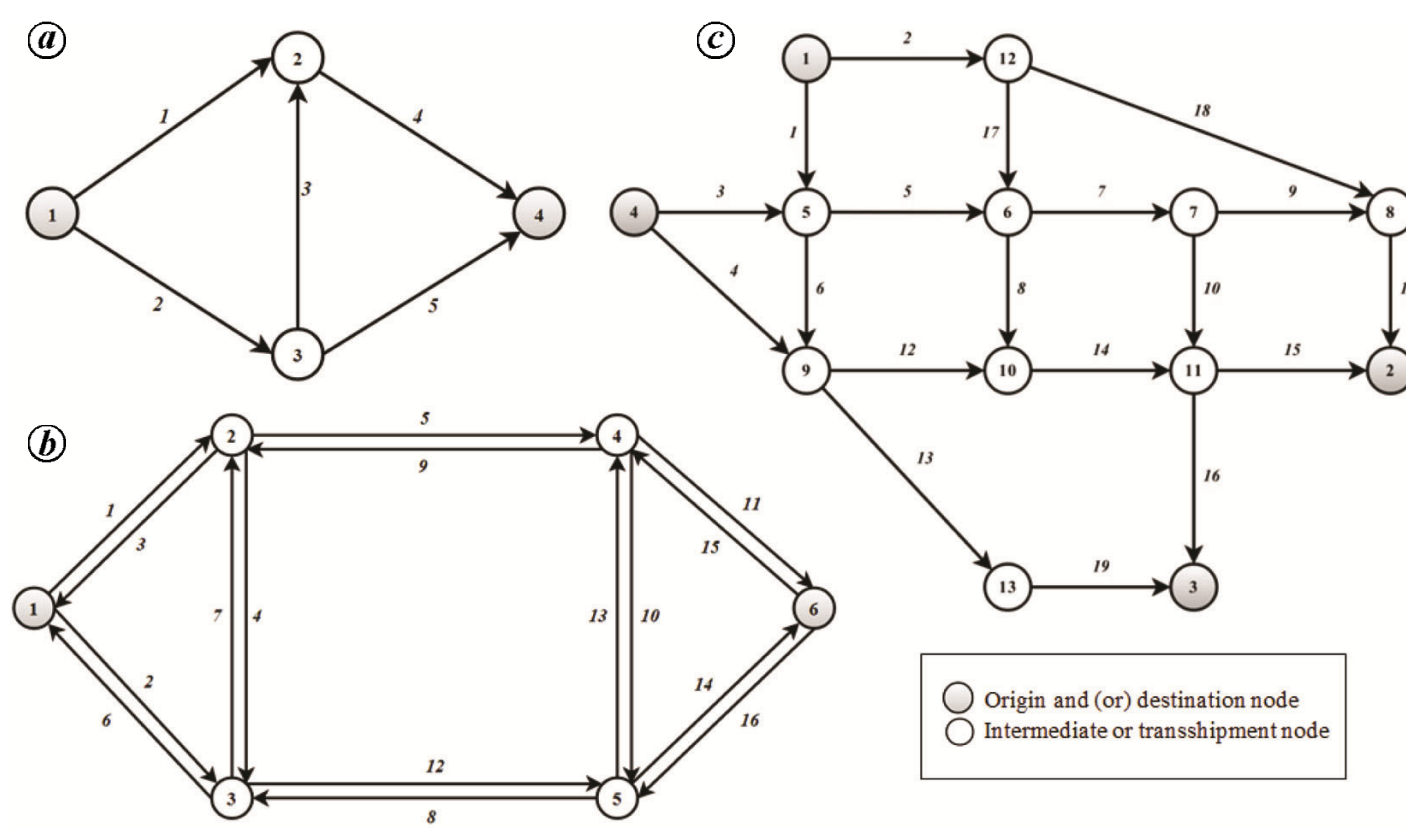

\section{Origin and (or) destination node Intermediate or transshipment node}

Figure 8. $\boldsymbol{a}-\boldsymbol{c}$, Asymmetric test networks 1-3.
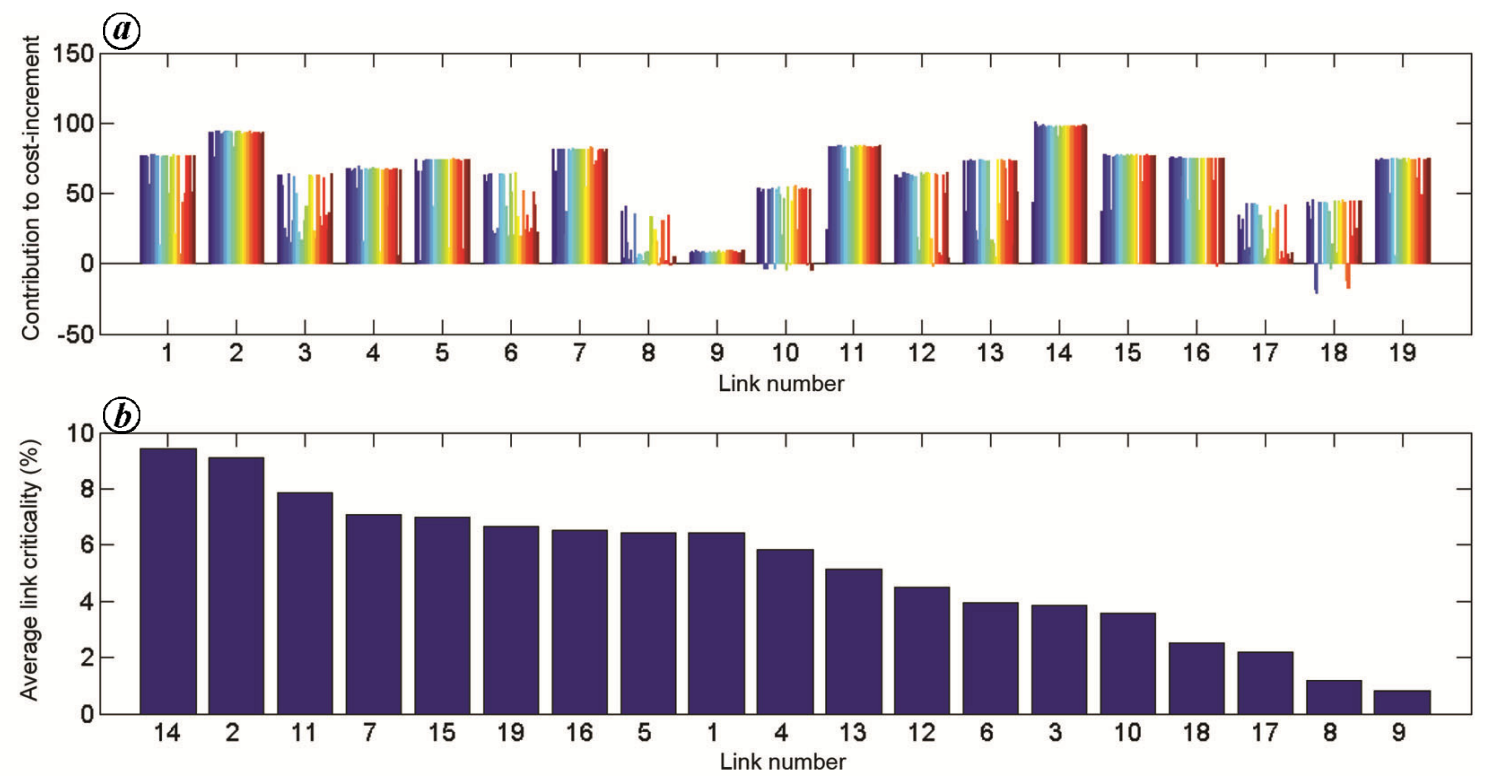

Figure 9. Critical links of the asymmetric test network 3. $\boldsymbol{a}$, Link contribution to cost increment. $\boldsymbol{b}$, critical links in descending order of rank.

Figure $9 b$ shows the average of the results, here the links are in the descending order of their criticality. In this example, the criticality ranking is similar, both with LC and $\overline{\mathrm{LC}}$. Figure 9 shows the results based on $\overline{\mathrm{LC}}$. Table 4 gives the order of criticality on the other two test networks.

\section{Sioux Falls network}

In this example, the topology and parameters of a network that relates to the road network at Sioux Falls, the largest city in the US state of South Dakota, are employed. The motivation behind selecting this network is that it is a widely cited benchmark for transportation network analysis. The network has 24 nodes, 76 links and 552 OD pairs.

We present two cases: one, a control experiment where the network is tested with a highly skewed OD matrix, and two, where the conventional OD matrix of the Sioux Falls network is adopted. In the former case, there exist only two OD pairs, i.e. the nodes 1 and 24 simultaneously act as origin and destination. Travel demand among 
Table 3. Link criticality on asymmetric test network 3

\begin{tabular}{rrrccc}
\hline Rank & $\begin{array}{c}\text { Link } \\
\text { number }\end{array}$ & $\begin{array}{c}\text { Percentage } \\
\text { degradation }\end{array}$ & $\begin{array}{c}\text { Contribution to STT } \\
\text { at zero degradation }\end{array}$ & $\begin{array}{c}\text { Contribution to STT } \\
\text { at critical state }\end{array}$ & $\begin{array}{c}\text { Link criticality } \\
\text { LC (\%) }\end{array}$ \\
\hline 1 & 14 & 70 & 3951 & 4049 & 9.9 \\
2 & 12 & 69 & 6212 & 6304 & 9.3 \\
3 & 11 & 75 & 4968 & 5051 & 8.3 \\
4 & 7 & 77 & 2532 & 2613 & 8.1 \\
5 & 1 & 79 & 3255 & 3332 & 7.5 \\
6 & 16 & 76 & 4188 & 4263 & 7.4 \\
7 & 5 & 78 & 1446 & 1520 & 7.4 \\
8 & 19 & 79 & 5133 & 5206 & 7.3 \\
9 & 13 & 79 & 4199 & 4272 & 6.3 \\
10 & 3 & 86 & 2766 & 2830 & 5.4 \\
11 & 4 & 72 & 7202 & 7256 & 5.3 \\
12 & 10 & 82 & 3471 & 3524 & 3.8 \\
13 & 15 & 72 & 4685 & 4723 & 3.5 \\
14 & 12 & 78 & 4240 & 4276 & 2.1 \\
15 & 6 & 40 & 2614 & 2635 & 1.2 \\
16 & 17 & 83 & 1811 & 1823 & 0.9 \\
17 & 8 & 85 & 3046 & 3055 & 0.8 \\
18 & 9 & 16 & 603 & 611 & -2.1 \\
19 & 18 & 9 & 6038 & 6017 & \\
& & & STT $_{\text {base }}=72,359$ & & \\
\end{tabular}
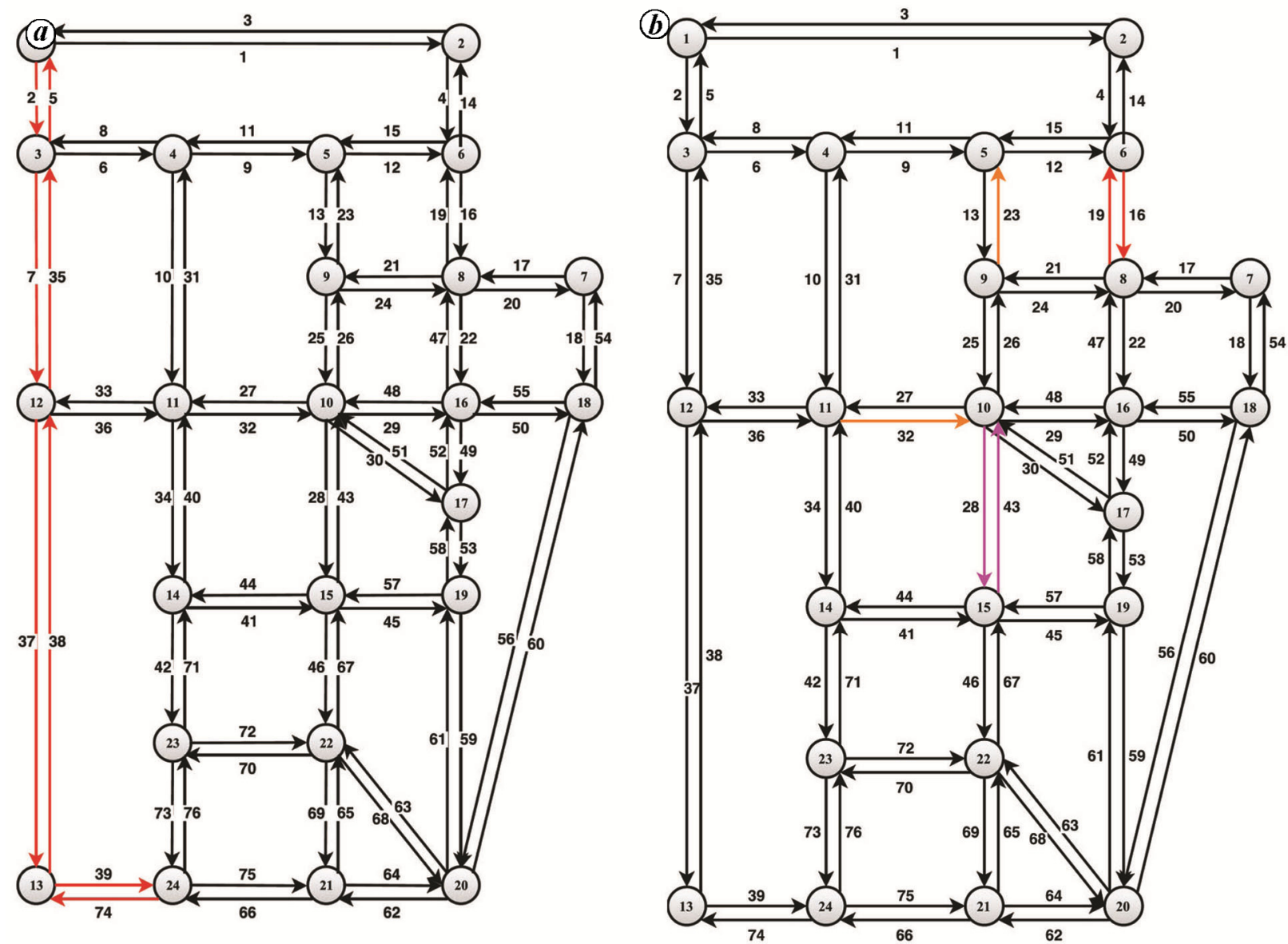

Figure 10. Critical links on the Sioux Falls network. Coloured links are more critical than the others. (a) Case with two OD pairs (1-24 and back). In $(\boldsymbol{a})$, the links in red $(2,5,7,35,37,38,39$ and 74$)$ are critical. (b) Case with 552 OD pairs. In $(\boldsymbol{b})$, the links in red (16 and 19) form a cluster of most critical links, followed by those in magenta (28 and 43), and those in orange (23 and 32 ). 
Table 4. Critical links of asymmetric test networks

\begin{tabular}{|c|c|c|c|}
\hline Network & Link no. & $\overline{\mathrm{LC}}$ & Criticality rank \\
\hline \multirow[t]{5}{*}{ Asymmetric test network 1} & 1 & 0.0 & 3 \\
\hline & 2 & 48.9 & 2 \\
\hline & 3 & 0.0 & 3 \\
\hline & 4 & 0.0 & 3 \\
\hline & 5 & 51.1 & 1 \\
\hline \multirow[t]{16}{*}{ Asymmetric test network 2} & 1 & 4.8 & 9 \\
\hline & 2 & 19.6 & 2 \\
\hline & 3 & -8.2 & 15 \\
\hline & 4 & -4.7 & 13 \\
\hline & 5 & 27.4 & 1 \\
\hline & 6 & 17.1 & 4 \\
\hline & 7 & -1.8 & 12 \\
\hline & 8 & 6.9 & 8 \\
\hline & 9 & 4.4 & 11 \\
\hline & 10 & -7.1 & 14 \\
\hline & 11 & 16.5 & 5 \\
\hline & 12 & 17.7 & 3 \\
\hline & 13 & -21.0 & 16 \\
\hline & 14 & 11.2 & 7 \\
\hline & 15 & 12.7 & 6 \\
\hline & 16 & 4.5 & 10 \\
\hline
\end{tabular}

all other OD pairs is set to zero. Figure $10 a$ shows the results. Note that all links from node 1 to node 24 , and back, are the most critical ones, probably because these links form the shortest path connecting the only nodes that face any travel demand.

On the other hand, the second case has 552 OD pairs. We present this to illustrate the relevance of the proposed method in a real-life scale. Figure $10 b$ shows the most critical links identified using this method. While prioritizing improvements in the network, a planner may use this information.

\section{Conclusion}

Unlike most hitherto studies on link criticality, the methodology presented here accounts for multiple simultaneous link capacity disruptions, thus addressing a relevant aspect, as most road networks of the world are subject to non-uniform capacity degradation on many of their links simultaneously. Simultaneous degradations on link-capacities result in many scenarios with different network costs. Employing a unique operable upper bound, we show that certain links contribute more to it, and hence are more critical than others on asymmetric networks. The methodology defines all links to be of comparable criticality on symmetric networks. This results agrees to natural intuition, and therefore imparts confidence to the methodology.

While identifying link criticality, this methodology considers travel demand of a normal day. It must be noted that criticality of a component can be different in case of catastrophes, evacuation or major events, where the demand may not be met. In various other cases - say while planning for investment, maintenance or operation - this measure could be incorporated to improve the cost-benefit analyses that planners employ for evaluating different options.

The approach in this study opens up interesting dimensions for future research. One dimension is about the retrofitting alternatives on the road network. Further studies could incorporate different response mechanisms and identify the changes in criticality of links dynamically over the period of a day. Such an inquiry can account for manifold concerns regarding routing, information gathering-cum-dissemination capability, efficacy of system-wide interventions, and the like.

1. Duke, C. B. et al., Network Science: Report from the Committee on Network Science for Future Army Applications, The National Academies Press, Washington, DC, 2007.

2. Newman, M. E., The structure and function of complex networks. SIAM Rev., 2003, 45, 167-256.

3. Nagurney, A. and Qiang, Q., Fragile networks: identifying vulnerabilities and synergies in an uncertain age. Int. Trans. Oper. Res., 2012, 19, 123-160.

4. Callaway, D. S., Newman, M. E., Strogatz, S. H. and Watts, D. J., Network robustness and fragility: percolation on random graphs. Phys. Rev. Lett., 2000, 85, 5468.

5. Balijepalli, C. and Oppong, O., Measuring vulnerability of road network considering the extent of serviceability of critical road links in urban areas. J. Transp. Geogr., 2014, 39, 145-155.

6. Hines, P., Cotilla-Sanchez, E. and Blumsack, S., Do topological models provide good information about electricity infrastructure vulnerability? Chaos: Interdiscip. J. Nonlinear Sci., 2010, 20, 033122.

7. Barabási, A. L., Linked: How Everything is Connected to Everything Else and What it Means for Business, Science, and Everyday life, Basic Books, New York, USA, 2014.

8. Scott, D. M., Novak, D. C., Aultman-Hall, L. and Guo, F., Network robustness index: a new method for identifying critical links and evaluating the performance of transportation networks. J. Transp. Geogr., 2006, 14, 215-227.

9. Sullivan, J. L., Novak, D. C., Aultman-Hall, L. and Scott, D. M., Identifying critical road segments and measuring system-wide robustness in transportation networks with isolating links: a linkbased capacity-reduction approach. Transp. Res. Part A, 2010, 44, 323-336.

10. Cats, O. and Jenelius, E., Planning for the unexpected: the value of reserve capacity for public transport network robustness. Transp. Res. Part A, 2015, 81, 47-61.

11. Taylor, M. A. and D'Este, G. M., Transport network vulnerability: a method for diagnosis of critical locations in transport infrastructure systems. In Critical Infrastructure - Reliability and Vulnerability (eds Murray, A. T. and Grubesic, T.), Springer, Berlin, Germany, 2007, pp. 9-30.

12. Nagurney, A. and Qiang, Q., A network efficiency measure with application to critical infrastructure networks. J. Global Optim., 2008, 40, 261-275.

13. Nagurney, A. and Qiang, Q., A transportation network efficiency measure that captures flows, behavior, and costs with applications to network component importance identification and vulnerability. In Proceedings of the POMS 18th Annual Conference, Dallas, Texas, USA, 2007.

14. Rodríguez-Núñez, E. and García-Palomares, J. C., Measuring the vulnerability of public transport networks. J. Transp. Geogr., 2014, 35, 50-63. 
15. Halder, A. K., The method of competing links. Transp. Sci., 1970 , 4, 36-51.

16. Sohn, J., Evaluating the significance of highway network links under the flood damage: an accessibility approach. Transp. Res. Part A, 2006, 40, 491-506.

17. Chen, B. Y., Lam, W. H., Sumalee, A., Li, Q. and Li, Z. C., Vulnerability analysis for large-scale and congested road networks with demand uncertainty. Transp. Res. Part A, 2012, 46, 501516.

18. Knoop, V. L., Snelder, M., van Zuylen, H. J. and Hoogendoorn, S. P., Link-level vulnerability indicators for real-world networks. Transp. Res. Part A, 2012, 46, 843-854.

19. Murray-Tuite, P. M. and Mahmassani, H. S., Methodology for determining vulnerable links in a transportation network. Transp. Res. Rec., 2004, 1882, 88-96.

20. Bell, M. G., Kanturska, U., Schmöcker, J. D. and Fonzone, A., Attacker-defender models and road network vulnerability. Philos. Trans. R. Soc. London, Ser. A, 2008, 366, 1893-1906.

21. Bhavathrathan, B. K. and Patil, G. R., Capacity uncertainty on urban road networks: A critical state and its applicability in resilience quantification. Comput. Environ. Urban Syst., 2015, 54, $108-118$.

22. Bhavathrathan, B. K. and Patil, G. R., Quantifying resilience using a unique critical cost on road networks subject to recurring capacity disruptions. Transportmetrica A: Transp. Sci., 2015, 11, 836-855.

23. Patil, G. R. and Bhavathrathan, B. K., Effect of traffic demand variation on road network resilience. Adv. Complex Syst., 2016, 19, 1650003.

24. Liberatore, F., Scaparra, M. P. and Daskin, M. S., Analysis of facility protection strategies against an uncertain number of attacks: The stochastic R-interdiction median problem with fortification. Comp. Oper. Res., 2011, 38, 357-366.

25. Zhuang, J. and Bier, V. M., Balancing terrorism and natural disasters - defensive strategy with endogenous attacker effort. Oper. Res., 2007, 55, 976-991.

26. Cappanera, P. and Scaparra, M. P., Optimal allocation of protective resources in shortest-path networks. Transp. Sci., 2011, 45, 64-80.
27. Fan, K., Minimax theorems. Proc. Natl. Acad. Sci. USA, 1953, 39, 42-47.

28. Parthasarathy, T. and Raghavan, T. E. S., Some Topics in TwoPerson Games, Elsevier Publishing Company, New York, 1971.

29. Brown, G. W., Iterative solution of games by fictitious play. Acta Anal. Prod. Alloc., 1951, 13, 374-376.

30. Robinson, J., An iterative method of solving a game. Ann. Math., 1951, 54, 296-301.

31. Sheffi, Y., Urban Transportation Networks: Equilibrium Analysis with Mathematical Programming Models, Prentice-Hall Inc., Englewood Cliffs, New Jersey, 1985.

32. Fishman, G. S., Discrete-Event Simulation: Modeling, Programming and Analysis, Springer Science \& Business Media, New York, 2013.

33. Bhavathrathan, B. K. and Patil, G. R., Algorithm to compute urban road network resilience. Transp. Res. Rec., 2018, 2672 , 104-115.

34. Fudenberg, D. and Levine, D., The Theory of Learning in Games, The MIT Press, Cambridge, MA, USA, 1998.

35. McMahan, H. B. and Gordony, G. J., A fast bundle-based anytime algorithm for poker and other convex games. In Proceedings of the Eleventh International Conference on Artificial Intelligence and Statistics, San Juan, Puerto Rico, 2007.

36. Harker, P. T. and Friesz, T. L., Bounding the solution of the continuous equilibrium network design problem. In Papers Presented during the Ninth International Symposium on Transportation and Traffic Theory, Delft, The Netherlands, 1984.

37. Nguyen, S. and Dupuis, C., An efficient method for computing traffic equilibria in networks with asymmetric transportation costs. Transp. Sci., 1984, 18, 185-202.

Received 27 June 2018; revised accepted 10 October 2019

doi: $10.18520 / \mathrm{cs} / \mathrm{v} 118 / \mathrm{i} 3 / 428-438$ 